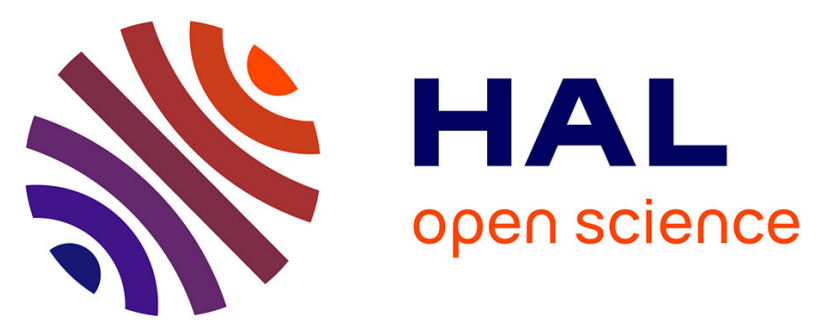

\title{
Correlating the Seebeck coefficient of thermoelectric polymer thin films to their charge transport mechanism
}

Ioannis Petsagkourakis, Eleni Pavlopoulou, Eric Cloutet, Yan Fang Chen, Xjianjie Liu, Mats Fahlman, Magnus Berggren, Xavier Crispin, Stefan Dilhaire, Guillaume Fleury, et al.

\section{To cite this version:}

Ioannis Petsagkourakis, Eleni Pavlopoulou, Eric Cloutet, Yan Fang Chen, Xjianjie Liu, et al.. Correlating the Seebeck coefficient of thermoelectric polymer thin films to their charge transport mechanism. Organic Electronics, 2018, 52, pp.335-341. 10.1016/j.orgel.2017.11.018 . hal-01649309

\section{HAL Id: hal-01649309 \\ https://hal.science/hal-01649309}

Submitted on 12 Dec 2017

HAL is a multi-disciplinary open access archive for the deposit and dissemination of scientific research documents, whether they are published or not. The documents may come from teaching and research institutions in France or abroad, or from public or private research centers.
L'archive ouverte pluridisciplinaire HAL, est destinée au dépôt et à la diffusion de documents scientifiques de niveau recherche, publiés ou non, émanant des établissements d'enseignement et de recherche français ou étrangers, des laboratoires publics ou privés.

\section{다(1)(2)}

Distributed under a Creative Commons Attribution - ShareAlikel 4.0 International 


\title{
Correlating the Seebeck coefficient of thermoelectric polymer thin films to their charge transport mechanism
}

\author{
Ioannis Petsagkourakis ${ }^{\mathrm{a}}$, Eleni Pavlopoulou, ${ }^{\mathrm{b}, * *}$, Eric Cloutet $^{\mathrm{a}}$, Yan Fang Chen ${ }^{\mathrm{c}}$, Xjianjie Liu ${ }^{\mathrm{d}}$, \\ Mats Fahlman ${ }^{\mathrm{d}}$, Magnus Berggren ${ }^{\mathrm{e}}$, Xavier Crispin ${ }^{\mathrm{e}}$, Stefan Dilhaire ${ }^{\mathrm{f}}$, Guillaume Fleury ${ }^{\mathrm{a}, *}$, \\ Georges Hadziioannou ${ }^{\mathrm{a}, * * *}$ \\ ${ }^{\mathrm{a}}$ Laboratoire de Chimie des Polymères Organiques (LCPO), CNRS UMR 5629 - Université de Bordeaux - ENSCBP, F-33607, Pessac, France \\ ${ }^{\mathrm{b}}$ Laboratoire de Chimie des Polymères Organiques (LCPO), Institut Polytechnique de Bordeaux (Bordeaux INP), F-33607, Pessac, France \\ ${ }^{\mathrm{c}}$ Laboratoire de l'Intégration du Matériau au Système, CNRS UMR 5218 - Bordeaux INP - Université de Bordeaux, F-33400, Talence, France \\ ${ }^{\mathrm{d}}$ Surface Physics and Chemistry, Department of Physics and Measurement Technology, Linköping University, SE-58183, Linköping, Sweden \\ ${ }^{\mathrm{e}}$ Laboratory of Organic Electronics, Department of Science and Technology, Linköping University, SE-60174, Norrköping, Sweden \\ ${ }^{\mathrm{f}}$ Laboratoire Ondes et Matière d'Aquitaine, CNRS UMR 5798 - Université de Bordeaux, F-33400, Talence, France
}

\section{A B S T R A C T}

Keywords:

Thermoelectrics

Conducting polymers PEDOT

Charge transport

Seebeck coefficient

\begin{abstract}
Room temperature flexible heat harvesters based on conducting polymers are ideally suited to cover the energy demands of the modern nomadic society. The optimization of their thermoelectric efficiency is usually sought by tuning the oxidation levels of the conducting polymers, even if such methodology is detrimental to the Seebeck coefficient $(S)$ as both the Seebeck coefficient and the electrical conductivity $(\sigma)$ are antagonistically related to the carrier concentration. Here we report a concurrent increase of $S$ and $\sigma$ and we experimentally derive the dependence of Seebeck coefficient on charge carrier mobility for the first time in organic electronics. Through specific control of the conducting polymer synthesis, we enabled the formation of a denser percolation network that facilitated the charge transport and the thermodiffusion of the charge carriers inside the conducting polymer layer, while the material shifted from a Fermi glass towards a semi-metal, as its crystallinity increased. This work sheds light upon the origin of the thermoelectric properties of conducting polymers, but also underlines the importance of enhanced charge carrier mobility for the design of efficient thermoelectric polymers.
\end{abstract}

\section{Introduction}

Organic thermoelectric materials based on poly(3,4 ethylenediox ythiophene) (PEDOT) conducting derivatives appear as a promising candidate for the development of low cost printed thermo generators for near room temperature applications [ 13 3]. Compared to their in organic counterparts, conducting polymers exhibit several advantages such as solution processability, material abundance and an inherent low thermal conductivity $[1,4]$. The energy conversion efficiency for ther moelectric materials can be defined using the figure of merit, $Z T=$ $\left(S^{2} \cdot \sigma \cdot T\right) / \kappa$, where $S$ is the Seebeck coefficient, $\sigma$, the electrical con ductivity, and $\kappa$, the thermal conductivity at a given temperature $T$ [4]. The optimization of the thermoelectric properties in such materials mainly focuses on the improvement of the power factor, $S^{2} \sigma$, as their low and mostly constant thermal conductivity $(0.20 .4 \mathrm{~W} / \mathrm{mK})$ already constitutes a strong asset [5 8]. As the Seebeck coefficient and electrical conductivity follow an antagonistic behavior with respect to the carrier concentration of the system, a proper tuning of the PEDOT doping level was shown to be crucial for enhanced thermoelectric properties $[4,5]$. Doping also affects the electrical conductivity, $\sigma$, since:

$\sigma=q \cdot N \cdot \mu$

where $q$ is the electric charge, $N$ the carrier concentration and $\mu$ the charge carrier mobility.

The carrier concentration is related to the filling (doping) and shape of the density of states (DoS) of the material [9 12$]$, while the charge carrier mobility is linked to the shape of the DoS for conducting poly mers for which the dominant charge transport mechanism is hopping $[12,13]$. On the other hand, following Mott's formalism, the Seebeck coefficient is related to conductivity through Equation (2) and, there fore, $S$ is also dependent on the DoS slope at the Fermi level, $E_{f}$; a steeper slope leading to a higher Seebeck coefficient $[14,15]$.

\footnotetext{
* Corresponding author.

** Corresponding author.

**** Corresponding author.

E-mail addresses: epavlopoulou@enscbp.fr (E. Pavlopoulou), gfleury@enscbp.fr (G. Fleury), hadzii@enscbp.fr (G. Hadziioannou).
} 
$S(E, T)=\frac{\pi^{2}}{3} \cdot \frac{k^{2} T}{q} \cdot\left(\frac{\partial \ln (\sigma(E))}{\partial E}\right)_{E=E_{f}}$

As evidenced by Equation (2), an appropriate tailoring of the DoS, and consequently of charge transport, is essential for improved ther moelectric properties. Yet, a dedicated experimental study of the effect of charge transport properties on the Seebeck coefficient of polymer thermoelectrics is still lacking.

Tailoring the DoS can be achieved through a suitable engineering of the PEDOT crystalline structure [6]. An enhanced PEDOT crystallinity assists carrier delocalization along the conjugation axis as well as im proves $\pi$ orbital overlapping along the $\pi$ stacking direction $[16,17]$. This has a direct effect on the electronic band structure of the material, notably on the Fermi level position and on the shape and filling of the DoS $[6,18]$, which, in turn, affects the charge transport properties. As a consequence, the electronic transition from a Fermi glass to a semi metallic behavior has been reported for PEDOT doped with $\mathrm{p}$ toluene sulfonate molecules (PEDOT:Tos) by Crispin and co workers [6] and the corresponding electronic structure was referred to as a bipolaron net work. Both electrical conductivity and Seebeck coefficient were posi tively affected following this approach.

The crystallinity of semi conducting polymer thin films is strongly dependent on the synthetic and processing parameters [19 23]. For instance, the addition of Dimethylsulfoxide (DMSO) or Ethylene Glycol (EG) in PEDOT formulations results in thin films of higher crystallinity and higher charge carrier mobility [22,24]. It is commonly accepted that these additives act as plasticizers for the PEDOT chains during the in situ polymerization leading to enhanced crystallinity as confirmed by grazing incidence $\mathrm{X}$ ray scattering experiments. Increasing the mole cular weight of conjugated polymers is a second lever which can lead to enhanced crystallinity [25 27], and, as a result, to improved electronic properties [28 30]. Consequently the control of the oxidative PEDOT polymerization is essential for optimal electronic and structural prop erties as the release of free protons during the process induces an ac celeration of the polymerization kinetics which is detrimental for chain length control [31]. The introduction of organic bases in PEDOT for mulations, such as pyridine, limits this auto catalyzed mechanism leading to higher molecular weight PEDOT chains with larger orbital delocalization along the backbone [29,30]. Meanwhile, higher mole cular weight chains could act as tie bridges between the crystallites of the system, forming a percolation network for the charge carriers, that enhances charge transport [32 34].

In this report we aim to study the relation between the Seebeck coefficient and the charge transport properties of PEDOT:Tos con ducting polymers. The charge carrier mobility is proven to be crucial for improving the Seebeck coefficient of inorganic components [35], pathway that is basically unexplored for pure polymeric systems. In order to achieve our goal, a series of PEDOT:Tos thin films that exhibit markedly different mobility values (expanding in a range as large as possible) was synthesized. Indeed, by engineering the structure of PEDOT:Tos following the two routes discussed in the previous para graph, we managed to obtain a wide range of charge carrier mobilities that enable us to extract a relationship between the Seebeck coefficient and $\mu$, while the charge carrier concentration is constant in all samples. It is highlighted that, to the best of our knowledge, no such relation has been reported so far in literature for conducting polymers. The origin of the interplay between $S, \mu$ and the underlying charge transport me chanisms of the materials are discussed, supported by ultraviolet pho toelectron spectra and temperature dependent electrical conductivity data.

\section{Experimental section}

\subsection{Synthesis of PEDOT:Tos thin films}

The $\mathrm{Fe}(\mathrm{Tos})_{3}$ oxidant solution (40\% in 1 butanol) was purchased from Heraus (Clevios B40), while EDOT, pyridine, DMSO, $\mathrm{n}$ butanol, ethanol were purchased from Sigma Aldrich. Poly(vinylidenefluoride co hexafluoropropylene) (P(VDF HFP)) and 1 ethyl 3 methylimidazo lium bis(trifluoromethylsulfonyl) amide ((EMI)(TFSA)) used for the fabrication of the electrolyte gated transistors were purchased from Sigma Aldrich. All materials were used as received. Pyridine was added in the $\mathrm{Fe}(\mathrm{Tos})_{3}$ oxidant solution in a ratio of $0.5 \mathrm{~mol}$ of pyridine to 1 mol of oxidant. DMSO was added in a volume fraction of $3 \%$ with respect to the oxidant solution volume. The oxidant/additives solutions were stirred for $12 \mathrm{~h}$ in ambient conditions and stored at $4{ }^{\circ} \mathrm{C}$ for subsequent use. The EDOT monomers were added to the Fe(Tos) ${ }_{3}$ oxi dant solution with an oxidant to monomer ratio equal to 2.3:1 [24,29], The resulting dispersions were spin coated on $15 \times 15 \mathrm{~mm}$ glass sub strates followed by a thermal annealing treatment at $100{ }^{\circ} \mathrm{C}$ during $15 \mathrm{~min}$ to initiate the polymerization reaction. Afterwards the films were sequentially washed with $\mathrm{n}$ butanol and ethanol, to remove the remaining oxidant, and dried under a nitrogen flow. The PEDOT:Tos films were further dried under vacuum overnight. Through the tuning of the EDOT dispersion spin coating conditions, PEDOT:Tos films with a typical thickness of $100 \pm 8 \mathrm{~nm}$ were obtained as determined using a stylus profilometer.

\subsection{Characterization methods}

Electrical conductivity was calculated as the inverse product of the Sheet resistance, $R_{\square}$, and the thickness, $t$. Sheet resistance was measured directly on the film surface via a 4 point probe set up utilizing a Lucas Lab S 3024 station. The film thickness was measured with a Dektak XT stylus profilometer. For the low temperature electrical conductivity measurement, a helium compressor in connection with a cryostat (Advanced research systems), and a Keithley 4200 source meter were used.

A homemade setup geometry $[36,37]$ was used to determine the Seebeck coefficient in thin film as the ratio between the thermovoltage, $V_{o c}$, under a temperature gradient $\Delta T$ (see Supporting Information).

GIWAXS measurements were performed on the Dutch Belgian Beamline (DUBBLE CRG), station BM26B, at the European Synchrotron Radiation Facility (ESRF), Grenoble, France [38]. The energy of the $\mathrm{X}$ rays was $12 \mathrm{keV}$, the sample to detector distance and the angle of in cidence, $\alpha_{i}$, were set at $11 \mathrm{~cm}$ and $0.16^{\circ}$, respectively. The diffracted intensity was recorded by a Frelon CCD camera and was normalized by the incident photon flux and the acquisition time (30 s). Flat field, polarization, solid angle and efficiency corrections were subsequently applied to the 2D GIWAXS images [39]. The scattering vector $q$ was defined with respect to the center of the incident beam and has a magnitude of $q=(4 \pi / \lambda) \sin (\theta)$, where $2 \theta$ is the scattering angle and $\lambda$ is the wavelength of the $\mathrm{X}$ ray beam. Herein we opted to present the wedge shaped corrected images (Fig. S1) where $q_{r}$ and $q_{z}$ are the in plane and near out of plane scattering vectors, respectively. The scat tering vectors are defined as follows: $q_{x}=(2 \pi / \lambda)\left(\cos \left(2 \theta_{\mathrm{f}}\right) \cos \left(\alpha_{\mathrm{f}}\right) \cos \right.$ $\left.\left(\alpha_{\mathrm{i}}\right)\right), \quad q_{\mathrm{y}}=(2 \pi / \lambda)\left(\sin \left(2 \theta_{\mathrm{f}}\right) \cos \left(\alpha_{\mathrm{f}}\right)\right), \quad q_{z}=(2 \pi / \lambda)\left(\sin \left(\alpha_{\mathrm{f}}\right)+\sin \left(\alpha_{\mathrm{i}}\right)\right)$, $q_{r}^{2}=q_{x}^{2}+q_{y}^{2}$, where $\alpha_{f}$ is the exit angle in the vertical direction and $2 \theta_{f}$ is the in plane scattering angle, in agreement with standard GI WAXS notation [40].

UPS measurements were carried out in a UHV surface analysis system equipped with a Scienta 200 hemispherical analyzer. The base pressure of a sample analysis chamber is $2 \times 10^{-8}$ mbar. UPS was performed using a standard He discharge lamp with $\mathrm{HeI} 21.22 \mathrm{eV}$ as excitation source and an energy resolution of $50 \mathrm{meV}$. 


\section{Results and discussion}

The PEDOT:Tos thin films were prepared by thermally activated in situ solution oxidative polymerization of EDOT monomers in the pre sence of $\mathrm{Fe}(\mathrm{Tos})_{3}$ (details are provided in the Experimental section) $[31,41]$. Different formulations of the EDOT solutions were employed in order to systematically increase the charge carrier mobility. Toluene (Tol), ethylene glycol (EG), dimethylformamide (DMF) and di methylsulfoxide (DMSO) have been used as additives, since such ad ditives act as plasticizers for the PEDOT chains during the in situ polymerization resulting in thin films of higher crystallinity, as already demonstrated in a previous report [24]. Additionally we have also de monstrated that the increase of crystallinity further translates into en hanced electrical conductivity and carrier mobility at a constant carrier concentration. All samples studied herein have been spin coated on glass substrates and the resulting film thickness was $100 \mathrm{~nm}$.

To further improve the charge carrier mobility of PEDOT:Tos, an organic base, that is pyridine, was introduced in the EDOT formulations in order to control the EDOT polymerization kinetics [29 31,41,42]. As underlined beforehand, the free protons that are released in the media during polymerization lead to an acceleration of the oxidative coupling reaction between the EDOT units. The introduction of organic bases inhibits this auto catalyzed mechanism allowing the synthesis of higher molecular weight PEDOT chains, with larger $\pi$ orbital delocalization [29]. Herein, we exploit the synergetic effect of the introduction of solvent additives and the presence of pyridine in the PEDOT formula tions in order to prepare PEDOT:Tos films which exhibit even higher charge carrier mobilities with respect to those reported in our previous work [24]. Table 1 lists the values of electrical conductivity, Seebeck coefficient and charge carrier mobility measured for the different PEDOT:Tos thin films synthesized by the addition of a high boiling point solvent or/and the addition of pyridine. The relative crystallinity of each film (defined as the ratio of the area of the (100) peak for the different systems with respect to that of the most crystalline system, i.e., that prepared with the addition of pyridine and DMSO in the for mulation) obtained from GIWAXS measurements is also reported in Table 1. The experimental procedure as well as details on the calcula tion of the relative crystallinity and the determination of $\mu, S$ and $\sigma$ are provided in the Experimental section as well as in the Supporting In formation (Figs. S1 S3). Note that the charge carrier mobility was de termined using electrolyte gated transistors under the assumption that the current passes homogeneously through the samples. This leads to an average effective mobility that is expected to underestimate the mobi lity but enables us to compare accurately the various PEDOT samples. The details on the device fabrication and characterization are provided

Table 1

The list of PEDOT:Tos thin films studied herein. The additives used in each formulation will be used through the manuscript as sample name. The conductivity, $\sigma$, Seebeck coefficient, $S$, mobility, $\mu$, and relative crystallinity values are provided along with their standard deviation. This was extracted by averaging the results obtained for 3 to 5 devices per case. The relative crystallinity of each film is defined as the ratio of the area of the (100) peak for the different systems with respect to that of the most crystalline system.

\begin{tabular}{|c|c|c|c|c|}
\hline Additives & $\sigma[\mathrm{S} / \mathrm{cm}]$ & $S[\mu \mathrm{V} / \mathrm{K}]$ & $\begin{array}{l}\mu \times 10^{4} \\
{\left[\mathrm{~cm}^{2} / \mathrm{Vs}\right]}\end{array}$ & $\begin{array}{l}\text { Relative } \\
\text { crystallinity }\end{array}$ \\
\hline No additive (Pristine) & $230 \pm 10$ & $32.0 \pm 5$ & $2.4 \pm 0.1$ & 0.21 \\
\hline Toluene (Tol) & $300 \pm 10$ & $32.6 \pm 5$ & $3.2 \pm 0.3$ & 0.33 \\
\hline Ethylene Glycol (EG) & $355 \pm 10$ & $34.0 \pm 5$ & $3.6 \pm 0.2$ & 0.39 \\
\hline $\begin{array}{l}\text { Dimethylformamide } \\
\text { (DMF) }\end{array}$ & $640 \pm 10$ & $35.1 \pm 5$ & $6.6 \pm 0.4$ & 0.42 \\
\hline $\begin{array}{l}\text { Dimethylsulfoxide } \\
\text { (DMSO) }\end{array}$ & $605 \pm 15$ & $32.2 \pm 5$ & $6.5 \pm 0.5$ & 0.41 \\
\hline Pyridine (Pyr) & $580 \pm 20$ & $40.0 \pm 2$ & $6.9 \pm 0.3$ & 0.56 \\
\hline Pyr + Tol & $600 \pm 20$ & $40.5 \pm 2$ & $7.0 \pm 0.3$ & 0.58 \\
\hline Pyr + EG & $890 \pm 70$ & $42.2 \pm 5$ & $9.8 \pm 0.2$ & 0.56 \\
\hline Pyr + DMF & $1225 \pm 40$ & $44.0 \pm 3$ & $12.8 \pm 0.9$ & 0.99 \\
\hline Pyr + DMSO & $1220 \pm 30$ & $44.0 \pm 2$ & $12.4 \pm 1$ & 1 \\
\hline
\end{tabular}

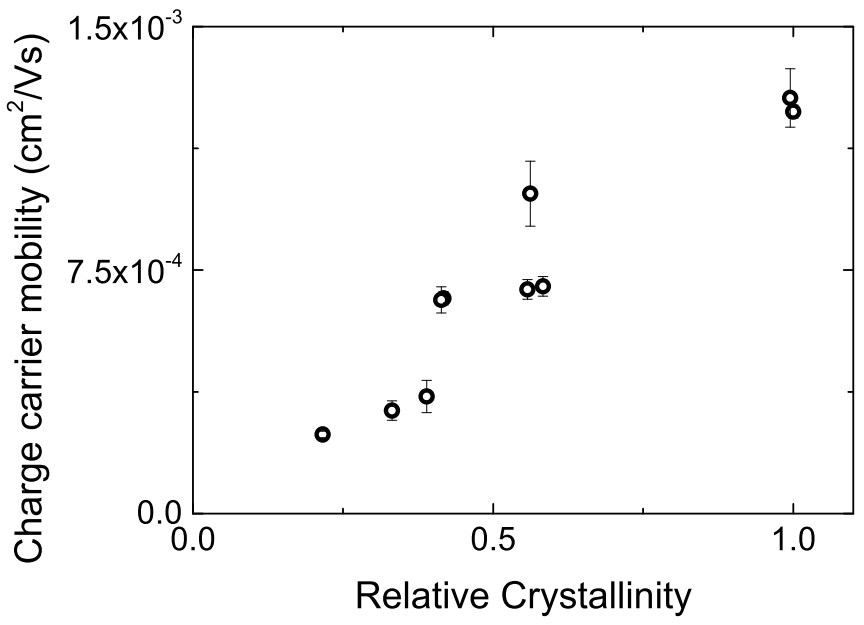

Fig. 1. Charge carrier mobility versus relative crystallinity for the PEDOT:Tos thin films.

in the Supporting Information and Fig. S3 shows the output char acteristics and transfer curves measured for these transistors.

As shown in Fig. 1, an enhanced crystallinity is highly beneficial to charge carrier mobility. This behavior is consistent and further extends our previous study on PEDOT:Tos systems, in which the strong inter play between crystallinity, crystallites orientation and charge transport properties was demonstrated [24]. We observe though, that the ex tracted values for the charge carrier mobility are relatively low (of the order of $\left.10^{-4} 10^{-3} \mathrm{~cm}^{2} / \mathrm{Vs}\right)$ considering the high oxidation level of PEDOT:Tos $(\approx 33 \%$ ) (Fig. S4) and the values already reported for such systems in the literature [43 45]. This discrepancy is related to the methodology used to determine the charge carrier mobility as we opted to work in an electrolyte gated Organic ElectroChemical Transistor (OECT) configuration, in consistence with the methodology proposed by Frisbie et coll. [46] and applied on PEDOT:PSS by Wei et al. [44]. In fact, preliminary measurements using a Field Effect Transistor (FET) configuration have demonstrated the inability to switch Off the FETs, invalidating this configuration to derive the charge carrier mobility of such highly conductive PEDOT:Tos systems. Additionally, during the operation of the OECT transistors, we clearly observed a dedoping of the material, similarly to the case reported by Wei et al. [44], which implies that charges transported throughout the bulk of the material contribute to the measurement. Consequently the reported $\mu$ values correspond to the mobility measured through the bulk of the material and not at the interface (i.e., the traditionally reported FET mobility). We recognize that our lower mobility with respect to the literature values originate from the different methodology followed for data analysis (OECT formalism in the low gate voltage regime) and we highlight that a dedicated study on measuring mobility in highly doped conjugated polymer systems should be conducted. Nevertheless, we can still carry out the analysis of the charge transport properties based on these mobility measurements, considering not the absolute values but rather their trend. Note that all trends reported below have been also verified by using the FET formalism, following the methodology of Wei et al. and similar behaviors were obtained. This allows us to plot the dependence of $\sigma$ on $\mu$ (see Fig. 2) where a linear correlation is observed. This behavior is characteristic of a constant charge carrier concentra tion, $N$, in all systems under study (see Equation (1)). This constant charge carrier concentration was further evaluated by the XPS spectra for our films, where the oxidation levels are shown to be constant and equal to 33\% (Fig. S4). Therefore, the filling of the DoS is unmodified upon the formulation conditions used herein [43].

The PEDOT:Tos films prepared with the pyridine containing for mulations that are examined herein provide the opportunity to extend the mobility range explored before by doubling the mobility values [24]. In Fig. 2 the Seebeck coefficient is plotted versus mobility. The 


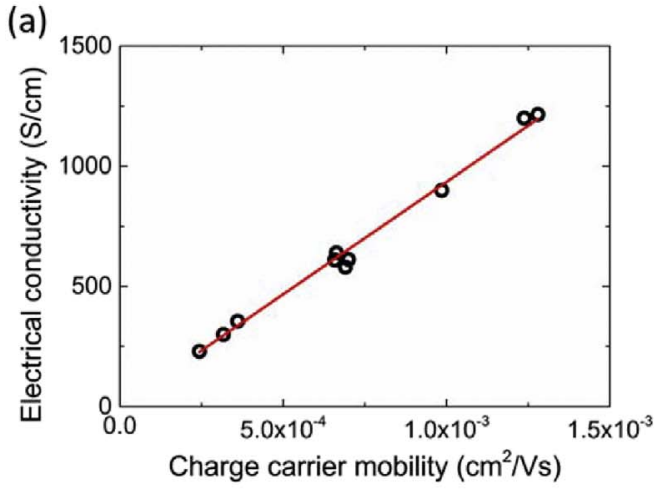

(b)

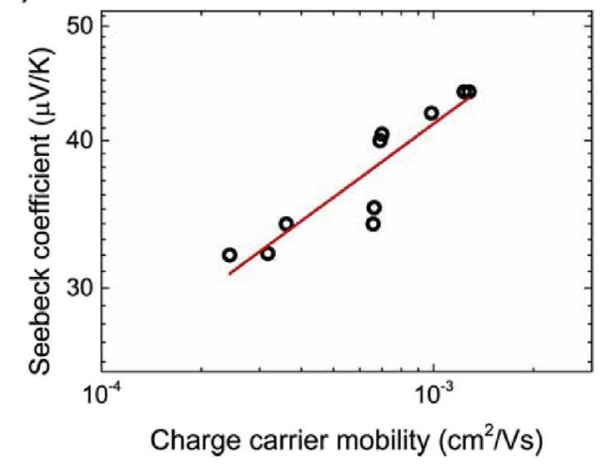

Fig. 2. (a) Electrical conductivity versus charge carrier mobility and (b) Seebeck coefficient versus charge carrier mobility for the PEDOT:Tos thin films under study. The red lines highlight: in (a), the direct proportionality between the electrical conductivity and the charge carrier mobility, which is consistent with Equation (1), and in (b), a weak dependence of the Seebeck coefficient with charge carrier mobility that follows a $S \propto$ $\mu^{0.2}$ power law. (For interpretation of the references to colour in this figure legend, the reader is referred to the web version of this article.) weak dependence between $S$ and $\mu$ is found to obey a $S \propto \mu^{0.2}$ power law. To the best of our knowledge this is the first time that a re lationship between Seebeck coefficient and charge carrier mobility is reported in literature for polymer semiconductors. Despite the weak dependence, it is worth exploring the origin of this behavior, since engineering the mobility of inorganic thermoelectrics is becoming in creasingly important, thanks to the alternative way for improving the Seebeck effect that this approach provides $[35,47]$.

In order to formalistically relate the Seebeck coefficient to charge carrier mobility, the energy dependent electrical conductivity, $\sigma(E)$, (valid at energies close to the Fermi position) should be considered (Equation (3)):

$\sigma(E)=q D(E) \mu(E)$

where $D(E)$ is the energy dependent density of states and $\mu(E)$ is the energy dependent charge carrier mobility [48].

The Mott's formalism on thermoelectricity (Equation (2)) can sub sequently be rewritten as the contribution of a "mobility dependent" term and a "DoS dependent" term as expressed in Equation (4).

$S=\frac{\pi^{2}}{3} \frac{k^{2} T}{q}\left(\frac{1}{\mu} \frac{\partial \mu}{\partial E}+\frac{1}{D} \frac{\partial D}{\partial E}\right)_{E_{f}}$

The "DoS dependent" term is related to the $D\left(E_{f}\right)$ and its slope at the Fermi level, $\left.\frac{\partial D}{\partial E}\right|_{E_{f}}$. Till recently the strategies employed in order to im prove $S$ aimed at maximizing this term, i.e. at engineering the electronic structure so as to increase the asymmetry of the $D(E)$ at the Fermi level $[6,35,47]$. Tuning the charge carrier concentration $N$ or tuning the structural order of the material could assist in this direction. The former stems from the fact that the charge carrier concentration is determined by the product $N=\int D(E) f(E) d E, f(E)$ being the Fermi Dirac dis tribution function [9]. The later has been demonstrated by Bubnova et al. [6], as an increase of the structural order has been shown to smooth out the DoS resulting in a steeper slope at the Fermi level. In our case, $N$ is constant for all samples, ruling out the effect of this para meter. However, the structural order increases, as witnessed by the increasing relative crystallinity.

To probe the effect of structural order on the DoS and its slope at the Fermi level, Ultraviolet Photoelectron Spectroscopy (UPS) experiments have been carried out. The full spectra that were recorded for four key PEDOT:Tos samples are presented in Fig. 3. The pristine PEDOT:Tos film as well as those prepared by the addition of DMSO, Pyridine and Pyridine + DMSO in the PEDOT formulations have been chosen, since they exhibit a gradually increasing crystallinity and mobility. The UPS spectra are in good agreement with previous reports on PEDOT:Tos films and the extracted work function value is $4.64 .8 \mathrm{eV}[6,49,50]$. Three distinct peaks one at $15.8 \mathrm{eV}$ related to the inelastic electron scattering due to the sample surface, and two at 9.5 and $6.1 \mathrm{eV}$ which are assigned to $\sigma$ states of the systems are apparent in the UPS spectra [49]. A zoom at the low binding energies is presented in Fig. 3b to allow the study of the DoS at the vicinity of the Fermi level. Frontier $\pi$ orbital states are probed in these energies and a broadening of the DoS as crystallinity increases is clearly noticed. This originates from the ex tended overlap of the $\pi$ orbitals $[1,6]$. It has been shown that this band edge broadening is transferred to all intermolecular $\pi$ bands, including those close to the Fermi position [51,52] and results in an enhanced charge carrier mobility and Seebeck coefficient [6]. Note that the in crease in mobility that is reported in Table 1 for these films perfectly correlates with the broadening of the DoS, the DMSO and pyridine samples having overlapping UPS spectra and similar mobility values. Yet, their DoS do not reflect their difference in crystallinity. The reason why will be discussed later. As far as the Seebeck coefficient is con cerned, a comparative look at Table 1 and Fig. 3, considering Equation (4), suggests that the change of slope of the DoS at the Fermi Level (schematically depicted in Fig. 3b by the dashed lines) is not consistent with the change in $S$ (the Pristine and DMSO treated PEDOT:Tos films have the same $S$, that gradually increases in case of the Pyridine and Pyridine + DMSO films). We conclude, thus, that the "DoS dependent" term in Mott's Equation (4) may indeed affect the Seebeck coefficient, as previously proposed [6], even if it is not the sole important para meter. The influence of the "mobility dependent" term should also be seriously considered. The "mobility dependent" term is related to the energy dependent relaxation time of the charge carriers, which physi cally originates from asymmetric scattering $[35,47]$. The contribution of this term has been ignored in most thermoelectric studies till now, one of the reasons being the strong dependence of Seebeck coefficient on the "Dos dependent" term, through its dependence on carrier con centration $[5,18]$. Nevertheless it becomes important for heavy fermion systems [47], and inorganic systems with large temperature dependent charge carrier mobility and concurrent low absolute mobility [35]. A close look at the data of that reference shows that a Variable Range Hopping (VRH) transport mechanism does not result in high $\frac{\partial \mu}{\partial T}$ (the gradient of mobility with respect to temperature, $\frac{\partial \mu}{\partial T}$, being physically equivalent to $\frac{\partial \mu}{\partial E}$ ), implying a limited contribution of the "mobility de pendent" term on Seebeck [35]. Yet, this is the mechanism that usually governs charge transport in polymer conducting materials, like those based on PEDOT. Consequently we studied the charge transport prop erties with respect to temperature in our systems in order to conclude on the effect of mobility on the thermoelectric properties.

Temperature dependent electrical conductivity measurements have been conducted and the results for the four PEDOT:Tos systems that have been already discussed with respect to their electronic properties are presented in Fig. 4. The electrical conductivity is presented nor malized with respect to the corresponding room temperature value. The electrical conductivity appears to be thermally activated for all PED OT:Tos systems under study. As crystallinity (i.e. order) increases, the normalized conductivity increases as well, in consistence with the current understanding on charge transport in disordered polymer semiconductors. In such systems charge transport mainly occurs through tunneling of the charge carriers between "metallic islands" (i.e. 
(a)

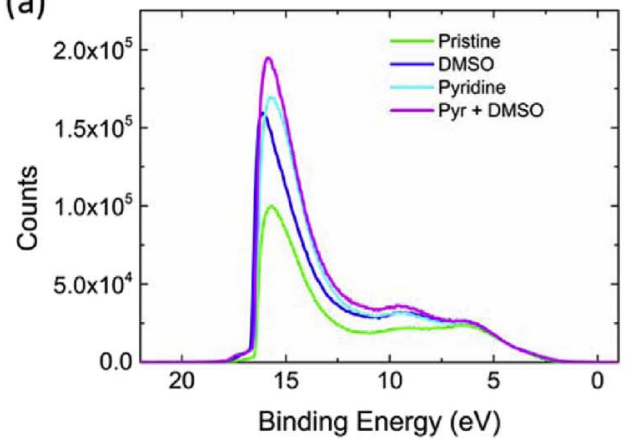

(b)

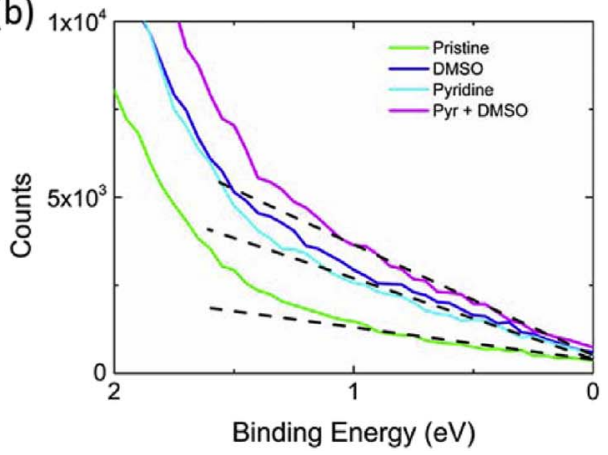

Fig. 3. (a) The UPS spectra for four representative PEDOT:Tos thin films with increasing crystallinity and mobility. (b) Magnified view of the lower binding energy region. The dashed lines represent the different slopes of the DoS at the Fermi level for the various samples. All spectra were normalized at $4.5 \mathrm{eV}$. doped PEDOT:Tos crystallites) dispersed in an amorphous matrix [53]. The more crystalline PEDOT:Tos thin films intrinsically contain less thermally activated traps, favoring charge carrier hopping between the "metallic" sites [53 56].

The Variable Range Hopping (VRH) model (Equation (5)) has been used to describe the transport in these PEDOT:Tos systems $[8,15,53,57,58]$,

$\sigma=\sigma_{0} \cdot e^{-\left(\frac{T_{0}}{T}\right)^{p}}$

where the pre factor $\sigma_{o}$ has a negligible dependence on $T, T_{O}$ is a hop ping parameter related to the DoS at the Fermi level and $p$ is a para meter related to the dimensionality, $d$, of the hopping transport, with $p=\frac{1}{1+d}$.

For $1 \mathrm{D}, 2 \mathrm{D}$ and $3 \mathrm{D}$ transport $p \approx 0.5,0.33$ and 0.25 , respectively [15]. The experimental data were fitted to Equation (5) (solid lines in Fig. 4a) and the parameters $\sigma_{o}, T_{o}$ and $p$ have been extracted (Table S1). The VRH model satisfactorily describes the behavior of the four systems under study. The temperature dependence of conductivity (and there fore of mobility (c.f. Equation (1)) is weak around room temperature, where we measure the thermoelectric properties. This temperature dependence of $\mu$ is much weaker than the $\mu \propto T^{7}$ reported for inorganics [35] and one would consider that it is not enough to induce a high "mobility dependent" contribution to the Seebeck coefficient (Equation (4)). Yet we cannot rule out its contribution, in particular given the non linearity between the DoS slope and the measured Seebeck values. Consequently we conclude that the observed dependence between $S$ and $\mu$ has to originate from the addition of small contributions from both terms of Equation (4). The weak $S \propto \mu^{0.2}$ dependence extracted from Fig. $2 b$ is consistent with small variations of these two terms for the studied PEDOT:Tos systems.

Further analysis of Fig. 4a allows us to explore the transport prop erties of these films. An interesting point concerns transport di mensionality. In fact $p$ shifts from 0.66 for the pristine and 0.59 for the DMSO treated sample, to 0.33 for the pyridine treated samples, which is translated to a transition from a quasi $1 \mathrm{D}$ transport to a $2 \mathrm{D}$ one. $\mathrm{Al}$ though, conducting polymers are effectively considered as 3D dis ordered metals, a quasi 1D or 2D dimensionality is observed, and it is assigned to the tunneling of charge carriers between mesoscopic "me tallic islands" inside the polymeric film [53,54]. Low conducting polymers with increased disordered are reported to exhibit high $p$ va lues $(\approx 0.5)$ that decrease to 0.25 as order and conductivity increase [54]. The films studied herein confirm this trend, since the more crystalline pyridine treated films exhibit a lower $p$. Moreover, the jump from $\approx 0.5$ for the non pyridine treated films to $\approx 0.33$ for the pyridine treated ones is explained by the higher molecular weight of PEDOT in the latter case that is induced by the proton scavenger role of pyridine during the polymerization of PEDOT $[29,30]$. As highlighted by Kline et al. [32] and Koch et al. [33], longer chains can assist the inter connection between crystallites in semiconducting polymers. These longer chains actually act as tie bridges between crystallites, forming an efficient percolation path between the "metallic islands" which pro motes charge transport and inhibits the scattering of charge carriers in the amorphous domains. That is the case in the pyridine treated PED OT:Tos films studied herein. The 2D transport that is evidenced in these samples is not assigned to the classical 2D transport, as described by Mott et al. [15], but rather to a network of interconnected "metallic islands" that offers multiple percolation paths for the transport of charge carriers. This finding puts forward the importance of a dense percolation network for efficient thermoelectric polymers. Indeed, while the DMSO and Pyridine treated samples appear to have similar UPS spectra and thus electronic properties, their Seebeck coefficients differ. This divergence could be attributed to the different transport dimensionalities that apply in the two samples. As Seebeck coefficient is directly related to the amount of charge carriers that are thermally diffused in system [4], a dense percolation network would promote the efficient transport of charge carriers, assisting, thus, their diffusion. Thus, a higher thermovoltage should be generated, as is the case for the pyridine treated films.

The temperature dependent conductivity measurements provide the possibility to further explore the charge transport properties by plotting the reduced activation energy, $W$, versus temperature, where $W=\frac{\partial \ln \sigma}{\partial \ln T}$ (Fig. 4b). The slope of $W$ is often used to characterize the conduction regime, as described by Mott's Metal to Insulator Transition (MIT) model for disordered semiconductors [16]. A positive slope corresponds
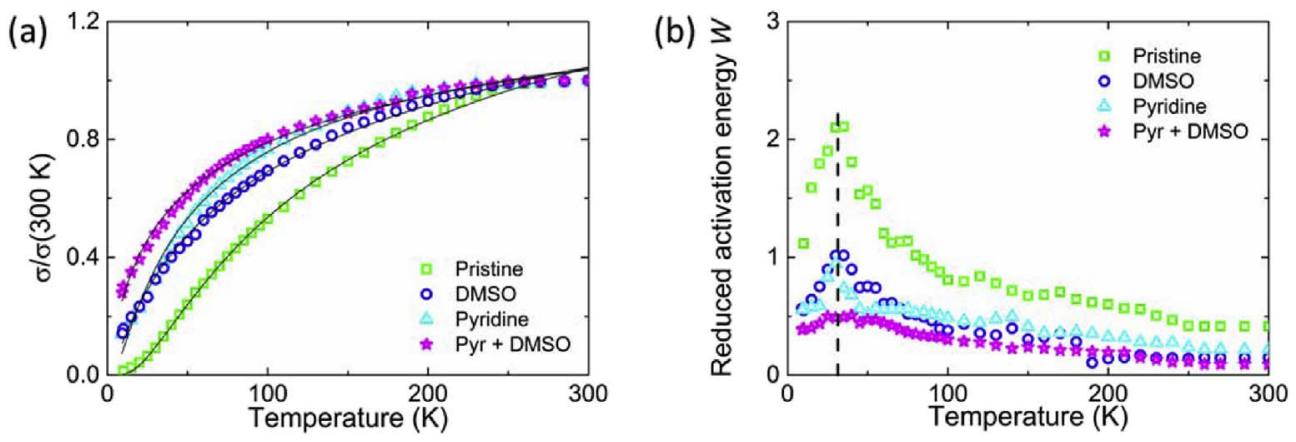

Fig. 4. (a) Normalized electrical conductivity versus temperature for the PEDOT:Tos thin films. The solid lines represent the fitting curves according to the VRH model. (b) Reduced activation energy, $W$, versus temperature for the PEDOT:Tos thin films. The dash line is a guide to the eyes for the metal-to-insulator transition at $30 \mathrm{~K}$. 
to a "metallic" regime, a negative to an "insulating" one while a slope equal to zero is related to the called "critical" regime. For all the PEDOT:Tos systems studied herein the slope of $W$ is positive below $30 \mathrm{~K}$, and negative above that value, tending to zero above $200 \mathrm{~K}$. Therefore a metal to insulator transition takes place at $30 \mathrm{~K}$, as found for other PEDOT derivatives [59]. The strength of this transition de creases as structural order increases in our systems. The appearance of a strong transition, i.e. of a pronounced conductivity maximum, has been related to the backscattering of charge carriers in the material [53]. This suggests that limited backscattering takes place in the Pyridine and Pyridine + DMSO treated films, which in fact is consistent with the improved percolation in these systems, as discussed previously. Above $200 \mathrm{~K}$, all systems under study tend towards a MIT critical regime, as the electrical conductivity is mostly constant at these high tempera tures. This is particularly true for the most crystalline one and is in agreement with the results reported for highly crystalline PEDOT:Tos films $[6,60,61]$. In that case a critical regime over a large range of temperatures without any visible transition has been demonstrated (note that the minimum probed temperature was $75 \mathrm{~K}$ ), and a semi metallic behavior has been identified. Therefore we are confident to suggest that at room temperature our materials stand between the pure semiconducting behavior usually observed for conjugated polymers [58] and the semi metallic behavior of a highly ordered bipolaron network [6]. We believe that the favorable percolation network that exists for all pyridine treated samples promotes the transition from a Fermi glass to a bipolaron network which is essential for high Seebeck coefficient organic thermoelectrics.

\section{Conclusion}

In this work, two processing methodologies have been combined to tune the structure of PEDOT:Tos and obtain a series of thin films within an extended mobility window. The thermoelectric properties of these films were studied and a weak $S \propto \mu^{0.2}$ dependence between the Seebeck coefficient and the charge carrier mobility, under constant carrier concentration, has been extracted. To our best knowledge this is the first time that such a relationship is reported for conducting polymers. The origin of this weak dependence was associated with two con tributions to the Seebeck coefficient; one originating from the electronic structure of the material as a broadening of the $\pi$ orbitals near the band edge was observed, and one originating from the charge transport characteristics apparent in these films. A transition from a quasi $1 \mathrm{D}$ to $2 \mathrm{D}$ hopping transport was observed with the gradual increase of thin film crystallinity, in particular for the pyridine treated samples that contain longer PEDOT chains. The later enabled the formation of a percolation network that is more efficient for charge transport as well as more beneficial for the thermoelectric properties of the polymer. Finally, a critical MIT regime is identified for our samples above $200 \mathrm{~K}$, which we believe is indicative of the transition between a semi conducting to a semi metallic behavior.

\section{Acknowledgements}

The authors acknowledge financial support from Arkema and the Région Aquitaine as well as from the Industrial Chair (Arkema/ANR) within the grant agreement no. AC 2013 365. I.P. is grateful to the Région Aquitaine for financial support (Ph.D. grant \#20111101004). The ESRF and NWO are acknowledged for allocating beam time at the Dutch Belgian beamline (DUBBLE) for the GIWAXS experiments. This work was performed within the framework of the Equipex ELORPrintTec ANR 10 EQPX 2801 and the Labex AMADEUS ANR 10 LABEX 0042 AMADEUS with the help of the French state Initiative d'Excellence IdEx ANR 10 IDEX 003 02. The Swedish contributions acknowledge the Knut and Alice Wallenberg foundation (Tail of the Sun) and the Advanced Functional Materials Center at Linköping University.

\section{References}

[1] O. Bubnova, X. Crispin, Towards polymer-based organic thermoelectric generators, Energy Environ. Sci. 5 (2012) 9345-9362, http://dx.doi.org/10.1039/c2ee22777k

[2] M. He, J. Ge, Z. Lin, X. Feng, X. Wang, H. Lu, Y. Yang, F. Qiu, Thermopower enhancement in conducting polymer nanocomposites via carrier energy scattering at the organic-inorganic semiconductor interface, Energy Environ. Sci. 5 (2012) 8351-8358, http://dx.doi.org/10.1039/c2ee21803h.

[3] Y. Chen, Y. Zhao, Z. Liang, Solution processed organic thermoelectrics: towards flexible thermoelectric modules, Energy Environ. Sci. 8 (2015) 401-422, http://dx doi.org/10.1039/C4EE03297G.

[4] G.J. Snyder, E.S. Toberer, Complex thermoelectric materials, Nat. Mater. 7 (2008) 105-114, http://dx.doi.org/10.1038/nmat2090.

[5] O. Bubnova, Z.U. Khan, A. Malti, S. Braun, M. Fahlman, M. Berggren, X. Crispin, Optimization of the thermoelectric figure of merit in the conducting polymer poly (3,4-ethylenedioxythiophene), Nat. Mater. 10 (2011) 429-433, http://dx.doi.org/ 10.1038/nmat3012.

[6] O. Bubnova, Z.U. Khan, H. Wang, S. Braun, D.R. Evans, M. Fabretto, P. HojatiTalemi, D. Dagnelund, J.-B. Arlin, Y.H. Geerts, S. Desbief, D.W. Breiby, J.W. Andreasen, R. Lazzaroni, W.M. Chen, I. Zozoulenko, M. Fahlman, P.J. Murphy, M. Berggren, X. Crispin, Semi-metallic polymers, Nat. Mater. 13 (2013) 190-194, http://dx.doi.org/10.1038/nmat3824.

[7] G.-H. Kim, L. Shao, K. Zhang, K.P. Pipe, Engineered doping of organic semiconductors for enhanced thermoelectric efficiency, Nat. Mater. 12 (2013) 719-723, http://dx.doi.org/10.1038/nmat3635.

[8] N. Massonnet, A. Carella, A. de Geyer, J. Faure-Vincent, J.-P. Simonato, Metallic behaviour of acid doped highly conductive polymers, Chem. Sci. 6 (2015) 412-417, http://dx.doi.org/10.1039/C4SC02463J.

[9] N.W. Ashcroft, N.D. Mermin, Solid State Physics, Holt, Rinehart and Winston, New York, 1976.

[10] G.P. Chen, Nanoscale Energy Transport and Conversion: a Parallel Treatment of Electrons, Molecules, Phonons, and Photons, Oxford University Press, Oxford, 2005

[11] M. Geoghegan, G. Hadziioannou, Polymer Electronics, Oxford University Press, Oxford, 2013, http://dx.doi.org/10.1093/acprof:oso/9780199533824.001.0001.

[12] V. Coropceanu, J. Cornil, D.A. da Silva Filho, Y. Olivier, R. Silbey, J.-L. Brédas, Charge transport in organic semiconductors, Chem. Rev. 107 (2007) 926-952, http://dx.doi.org/10.1021/cr050140x.

[13] R.P. Fornari, P.W.M. Blom, A. Troisi, How many parameters actually affect the mobility of conjugated polymers? Phys. Rev. Lett. 118 (2017) 86601, http://dx.doi. org/10.1103/PhysRevLett.118.086601.

[14] M. Cutler, N.F. Mott, Observation of Anderson localization in an electron gas, Phys. Rev. 181 (1969) 1336-1340, http://dx.doi.org/10.1103/PhysRev.181.1336.

[15] N.F. Mott, E.A. Davis, Electronic Processes in Non-crystalline Materials, 2d ed., Clarendon Press, Oxford;New York, 1979.

[16] N. Kim, B.H. Lee, D. Choi, G. Kim, H. Kim, J.-R. Kim, J. Lee, Y.H. Kahng, K. Lee, Role of interchain coupling in the metallic state of conducting polymers, Phys. Rev. Lett. 109 (2012) 106405, http://dx.doi.org/10.1103/PhysRevLett.109.106405.

[17] B.B.-Y. Hsu, C.-M. Cheng, C. Luo, S.N. Patel, C. Zhong, H. Sun, J. Sherman, B.H. Lee, L. Ying, M. Wang, G. Bazan, M. Chabinyc, J.-L. Brédas, A. Heeger, The density of states and the transport effective mass in a highly oriented semiconducting polymer: electronic delocalization in 1D, Adv. Mater. 27 (2015) 7759-7765, http:// dx.doi.org/10.1002/adma.201502820.

[18] S. Ihnatsenka, X. Crispin, I.V. Zozoulenko, Understanding hopping transport and thermoelectric properties of conducting polymers, Phys. Rev. B 92 (2015) 35201, http://dx.doi.org/10.1103/PhysRevB.92.035201.

[19] B.W. Boudouris, V. Ho, L.H. Jimison, M.F. Toney, A. Salleo, R.A. Segalman, Realtime observation of poly(3-alkylthiophene) crystallization and correlation with transient optoelectronic properties, Macromolecules 44 (2011) 6653-6658, http:// dx.doi.org/10.1021/ma201316a.

[20] D. Deribew, E. Pavlopoulou, G. Fleury, C. Nicolet, C. Renaud, S.-J. Mougnier, L. Vignau, E. Cloutet, C. Brochon, F. Cousin, G. Portale, M. Geoghegan, G. Hadziioannou, Crystallization-Driven enhancement in photovoltaic performance through block copolymer incorporation into P3HT: PCBM blends, Macromolecules 46 (2013) 3015-3024, http://dx.doi.org/10.1021/ma302128h.

[21] C.H. Woo, C. Piliego, T.W. Holcombe, M.F. Toney, J.M.J. Fréchet, A quantitative correlation between the mobility and crystallinity of photo-cross-linkable P3HT, Macromolecules 45 (2012) 3057-3062, http://dx.doi.org/10.1021/ma202203z.

[22] C.M. Palumbiny, F. Liu, T.P. Russell, A. Hexemer, C. Wang, P. Müller-Buschbaum, The crystallization of PEDOT: PSS polymeric electrodes probed in situ during printing, Adv. Mater. 27 (2015) 3391-3397, http://dx.doi.org/10.1002/adma 201500315.

[23] J.T. Rogers, K. Schmidt, M.F. Toney, G.C. Bazan, E.J. Kramer, Time-resolved structural evolution of additive-processed bulk heterojunction solar cells, J. Am. Chem. Soc. 134 (2012) 2884-2887, http://dx.doi.org/10.1021/ja2104747.

[24] I. Petsagkourakis, E. Pavlopoulou, G. Portale, B.A. Kuropatwa, S. Dilhaire, G. Fleury, G. Hadziioannou, Structurally-driven enhancement of thermoelectric properties within poly(3,4-ethylenedioxythiophene) thin films, Sci. Rep. 6 (2016) 30501, http://dx.doi.org/10.1038/srep30501.

[25] B. Endrődi, J. Mellár, Z. Gingl, C. Visy, C. Janáky, Molecular and supramolecular parameters dictating the thermoelectric performance of conducting polymers: a case study using poly(3-alkylthiophene)s, J. Phys. Chem. C 119 (2015) 8472-8479, http://dx.doi.org/10.1021/acs.jpcc.5b00135. 
[26] C. Goh, R.J. Kline, M.D. McGehee, E.N. Kadnikova, J.M.J. Fréchet, Molecularweight-dependent mobilities in regioregular poly(3-hexyl-thiophene) diodes, Appl. Phys. Lett. 86 (2005) 122110, http://dx.doi.org/10.1063/1.1891301.

[27] A. Zen, M. Saphiannikova, D. Neher, J. Grenzer, S. Grigorian, U. Pietsch, U. Asawapirom, S. Janietz, U. Scherf, I. Lieberwirth, G. Wegner, Effect of molecular weight on the structure and crystallinity of poly(3-hexylthiophene), Macromolecules 39 (2006) 2162-2171, http://dx.doi.org/10.1021/ma0521349.

[28] G. Juška, K. Genevičius, K. Arlauskas, R. Österbacka, H. Stubb, Features of charge carrier concentration and mobility in $\pi$-conjugated polymers, Macromol. Symp. 212 (2004) 209-218, http://dx.doi.org/10.1002/masy.200450820.

[29] Y.-H. Ha, N. Nikolov, S.K. Pollack, J. Mastrangelo, B.D. Martin, R. Shashidhar, Towards a transparent, highly conductive poly(3,4-ethylenedioxythiophene), Adv. Funct. Mater. 14 (2004) 615-622, http://dx.doi.org/10.1002/adfm.200305059.

[30] J.-H. Huang, C.-W. Chu, Achieving efficient poly(3,4-ethylenedioxythiophene)based supercapacitors by controlling the polymerization kinetics, Electrochim. Acta 56 (2011) 7228-7234, http://dx.doi.org/10.1016/j.electacta.2011.03.044.

[31] B. Winther-Jensen, D.W. Breiby, K. West, Base inhibited oxidative polymerization of 3,4-ethylenedioxythiophene with iron(III)tosylate, Synth. Met. 152 (2005) 1-4, http://dx.doi.org/10.1016/j.synthmet.2005.07.085.

[32] R. Joseph Kline, M.D. McGehee, M.F. Toney, Highly oriented crystals at the buried interface in polythiophene thin-film transistors, Nat. Mater. 5 (2006) 222-228, http://dx.doi.org/10.1038/nmat1590.

[33] F.P.V. Koch, J. Rivnay, S. Foster, C. Müller, J.M. Downing, E. Buchaca-Domingo, P. Westacott, L. Yu, M. Yuan, M. Baklar, Z. Fei, C. Luscombe, M.A. McLachlan, M. Heeney, G. Rumbles, C. Silva, A. Salleo, J. Nelson, P. Smith, N. Stingelin, The impact of molecular weight on microstructure and charge transport in semicrystalline polymer semiconductors-poly(3-hexylthiophene), a model study, Prog. Polym. Sci. 38 (2013) 1978-1989, http://dx.doi.org/10.1016/j.progpolymsci. 2013.07.009.

[34] R. Noriega, J. Rivnay, K. Vandewal, F.P.V. Koch, N. Stingelin, P. Smith, M.F. Toney, A. Salleo, A general relationship between disorder, aggregation and charge transport in conjugated polymers, Nat. Mater. 12 (2013) 1038-1044, http://dx.doi.org/ 10.1038/nmat3722.

[35] P. Sun, B. Wei, J. Zhang, J.M. Tomczak, A.M. Strydom, M. Søndergaard, B.B. Iversen, F. Steglich, G.D. Mahan, J.O. Sofo, J.R. Sootsman, D.Y. Chung, M.G. Kanatzidis, G.J. Snyder, E.S. Toberer, B.C. Sales, D. Mandrus, R.K. Williams, J.P. Heremans, I. Terasaki, Y. Sasago, H. Uchinokura, V. Zlatić, K. Behnia, Y. Wang, P. Sun, F. Steglich, J.S. Dyck, W. Chen, J. Yang, G.P. Meisner, C. Uher, E.H. Sondheimer, H. Liu, C. Xiao, A.M. Strydom, Large Seebeck effect by chargemobility engineering, Nat. Commun. 6 (2015) 7475, http://dx.doi.org/10.1038/ ncomms8475.

[36] J. Wüsten, K. Potje-Kamloth, Organic thermogenerators for energy autarkic systems on flexible substrates, J. Phys. D. Appl. Phys. 41 (2008) 135113, http://dx.doi.org/ 10.1088/0022-3727/41/13/135113.

[37] S. van Reenen, M. Kemerink, Correcting for contact geometry in Seebeck coefficient measurements of thin film devices, Org. Electron. 15 (2014) 2250-2255, http://dx. doi.org/10.1016/j.orgel.2014.06.018.

[38] G. Portale, D. Hermida-Merino, W. Bras, Polymer research and synchrotron radiation perspectives, Eur. Polym. J. 81 (2016) 415-432, http://dx.doi.org/10.1016/j. eurpolymj.2016.04.015.

[39] P. Müller-Buschbaum, The active layer morphology of organic solar cells probed with grazing incidence scattering techniques, Adv. Mater. 26 (2014) 7692-7709, http://dx.doi.org/10.1002/adma.201304187.

[40] G. Renaud, R. Lazzari, F. Leroy, Probing surface and interface morphology with grazing incidence small angle X-ray scattering, Surf. Sci. Rep. 64 (2009) 255-380, http://dx.doi.org/10.1016/j.surfrep.2009.07.002.

[41] W. Lövenich, PEDOT-properties and applications, Polym. Sci. Ser. C 56 (2014) 135-143, http://dx.doi.org/10.1134/S1811238214010068.

[42] A. Elschner, S. Kirchmeyer, W. Lovenich, U. Merker, K. Reuter, PEDOT: Principles and Applications of an Intrinsically Conductive Polymer, CRC Press, 2010, http://dx.doi.org/10.1201/b10318-10.
[43] B. Winther-Jensen, M. Forsyth, K. West, J.W. Andreasen, G. Wallace, D.R. MacFarlane, High current density and drift velocity in templated conducting polymers, Org. Electron. 8 (2007) 796-800, http://dx.doi.org/10.1016/j.orgel 2007.07.009.

[44] Q. Wei, M. Mukaida, Y. Naitoh, T. Ishida, Morphological change and mobility enhancement in PEDOT: PSS by adding co-solvents, Adv. Mater. 25 (2013) 2831-2836, http://dx.doi.org/10.1002/adma.201205158.

[45] S. Dongmin Kang, G. Jeffrey Snyder, Charge-transport model for conducting polymers, Nat. Mater. 16 (2016) 252-257, http://dx.doi.org/10.1038/nmat4784.

[46] K.H. Lee, M.S. Kang, S. Zhang, Y. Gu, T.P. Lodge, C.D. Frisbie, "Cut and stick" rubbery ion gels as high capacitance gate dielectrics, Adv. Mater. 24 (2012) 4457-4462, http://dx.doi.org/10.1002/adma.201200950.

[47] P. Sun, F. Steglich, Nernst effect: evidence of local Kondo scattering in heavy fermions, Phys. Rev. Lett. 110 (2013) 216408, http://dx.doi.org/10.1103/ PhysRevLett.110.216408.

[48] C. Kittel, Introduction to Solid State Physics, eighth ed., Wiley, 2005.

[49] K.Z. Xing, M. Fahlman, X.W. Chen, O. Inganäs, W.R. Salaneck, The electronic structure of poly(3,4-ethylene-dioxythiophene): studied by XPS and UPS, Synth Met. 89 (1997) 161-165, http://dx.doi.org/10.1016/S0379-6779(97)81212-X.

[50] L. Lindell, A. Burquel, F.L.E. Jakobsson, V. Lemaur, M. Berggren, R. Lazzaroni, J. Cornil, W.R. Salaneck, X. Crispin, Transparent, plastic, low-work-function poly (3,4-ethylenedioxythiophene) electrodes, Chem. Mater. 18 (2006) 4246-4252, http://dx.doi.org/10.1021/cm061081m.

[51] G. Koller, S. Berkebile, M. Oehzelt, P. Puschnig, C. Ambrosch-Draxl, F.P. Netzer, M.G. Ramsey, Intra- and intermolecular band dispersion in an organic crystal, Science 317 (2007) 351-355, http://dx.doi.org/10.1126/science.1143239.

[52] X. Crispin, J. Cornil, R. Friedlein, K.K. Okudaira, V. Lemaur, A. Crispin, G. Kestemont, M. Lehmann, M. Fahlman, R. Lazzaroni, Y. Geerts, G. Wendin, N. Ueno, J.-L. Brédas, W.R. Salaneck, Electronic delocalization in discotic liquid crystals: a joint experimental and theoretical study, J. Am. Chem. Soc. 126 (2004) 11889-11899, http://dx.doi.org/10.1021/ja048669j.

[53] A.B. Kaiser, V. Skákalová, Electronic conduction in polymers, carbon nanotubes and graphene, Chem. Soc. Rev. 40 (2011) 3786, http://dx.doi.org/10.1039/ c0cs00103a.

[54] A.B. Kaiser, G.U. Flanagan, D.M. Stewart, D. Beaglehole, Heterogeneous model for conduction in conducting polymers and carbon nanotubes, Synth. Met. 117 (2001) 67-73, http://dx.doi.org/10.1016/S0379-6779(00)00540-3.

[55] R. Menon, C.O. Yoon, D. Moses, A.J. Heeger, Y. Cao, Transport in polyaniline near the critical regime of the metal-insulator transition, Phys. Rev. B 48 (1993) 17685-17694, http://dx.doi.org/10.1103/PhysRevB.48.17685.

[56] K. Lee, S. Cho, S. Heum Park, A.J. Heeger, C.-W. Lee, S.-H. Lee, Metallic transport in polyaniline, Nature 441 (2006) 65-68, http://dx.doi.org/10.1038/nature04705.

[57] C.S.S. Sangeeth, M. Jaiswal, R. Menon, Charge transport in transparent conductors: a comparison, J. Appl. Phys. 105 (2009) 63713, http://dx.doi.org/10.1063/1. 3097759.

[58] A.J. Kronemeijer, E.H. Huisman, I. Katsouras, P.A. van Hal, T.C.T. Geuns, P.W.M. Blom, S.J. van der Molen, D.M. de Leeuw, Universal scaling in highly doped conducting polymer films, Phys. Rev. Lett. 105 (2010) 156604, http://dx.doi.org/ 10.1103/PhysRevLett.105.156604.

[59] R. Kiebooms, A. Aleshin, K. Hutchison, F. Wudl, Thermal and electromagnetic behavior of doped poly(3,4-ethylenedioxythiophene) films, J. Phys. Chem. B 101 (1997) 11037-11039, http://dx.doi.org/10.1021/jp9720101.

[60] J. Wang, K. Cai, S. Shen, Enhanced thermoelectric properties of poly(3,4-ethylenedioxythiophene) thin films treated with $\mathrm{H} 2$ SO 4, Org. Electron 15 (2014) 3087-3095, http://dx.doi.org/10.1016/j.orgel.2014.09.012.

[61] J. Wang, K. Cai, S. Shen, A facile chemical reduction approach for effectively tuning thermoelectric properties of PEDOT films, Org. Electron. 17 (2015) 151-158, http://dx.doi.org/10.1016/j.orgel.2014.12.007. 